$16^{\text {th }}$ International Congress of Metrology, 05005 (2013)

DOI: $10.1051 /$ metrology/201305005

(C) Owned by the authors, published by EDP Sciences, 2013

\title{
Comment adapter la métrologie à un laboratoire de biologie médicale ?
}

\author{
Loïc ROSO \\ Centre Technologique Méditerranéen de Métrologie (CT2M), Centre des creusets, Route de Lançon, 13250 Saint-Chamas
}

CT M!

\begin{abstract}
In the state of official texts, the biology laboratories must be accredited in the standard ISO 15189 [1] before the end of the year 2016 for the half of the acts they realize. Beyond the quality aspect this standard highlights the metrological requirements. Those requirements have led medical laboratory to formalize their needs and to ask the question of the internalization of the metrology, or at least of the internalization of the process of calibration. If the use of a subcontractor may look like an easy solution, the decision to internalize calibrations depends above all on the situation of the laboratory: number of instruments to be calibrated, characteristics of instruments, technicality of the procedure of calibration, cost, duration of immobilization ...
\end{abstract}

For some instruments, the lack of accredited laboratory has incited certain medical laboratories to acquire in intern the skill of the calibration. The necessity of an adapted metrology to every laboratory is thus a fact. So how to proceed? For example is it better to calibrate all laboratory pipettes to avoid the risk of misuse? Or is it better to doubt of the current organization : separate the critical pipettes, attribute to a laboratory sector, and even dedicate some pipettes to an analysis in particular ... This kind of questions is put. The questioning of the current organization is necessary and different responses have already been made. The metrology adapted to the biology: the challenge is started with the key of assurance of a better quality and reliability of the announced results.

Résumé. En l'état des textes officiels, les laboratoires de biologie médicale doivent être accrédités à la norme ISO 15189 [1] avant la fin de l'année 2016 pour la moitié des examens qu'ils réalisent. Une norme qui au-delà de l'aspect qualité met en avant les exigences métrologiques. Ces dernières ont donc emmené les laboratoires de biologie à formaliser leurs besoins et à se poser la question de l'internalisation de la fonction métrologie, ou du moins de l'internalisation du processus d'étalonnage. Si le recours à un sous-traitant peut apparaître comme une solution de facilité, la décision d'internaliser les étalonnages dépend avant tout de la situation du laboratoire: nombre d'instruments à étalonner, type d'instruments, technicité de la procédure d'étalonnage, coût, délai d'immobilisation...

Pour certains instruments, le manque de prestataire accrédité a incité certains laboratoires à acquérir en interne la compétence de l'étalonnage. La nécessité d'une métrologie adaptée à chaque laboratoire est donc un fait. Alors comment procéder? Par exemple, vautil mieux étalonner toutes les pipettes du laboratoire pour éviter les risques de mauvaise utilisation? Ou faut-il préférer remettre en cause l'organisation actuelle? Distinguer des pipettes critiques, les attribuer à un secteur du laboratoire, et même dédier certaines pipettes à une analyse en particulier ... Les question de ce type sont nombreuses. La remise en cause de l'organisation actuelle est nécessaire et différentes réponses ont déjà été apportées. Une métrologie adaptée à la biologie: le défi est lancé avec à la clef l'assurance d'une meilleure qualité et fiabilité des résultats annoncés. 


\section{POURQUOI DE LA MÉTROLOGIE EN BIOLOGIE MÉDICALE ?}

\begin{abstract}
Au fil des années, les publications [2], [3], [4] ont montré l'évolution des pratiques, et mis en évidence la nécessité de la métrologie pour assurer des résultats les plus fiables possibles. Améliorer l'exactitude permet notamment de diminuer les coûts, dus par exemple à des erreurs de diagnostic et/ou de mauvais traitement. La mise en place de la métrologie dans les laboratoires de biologie médicale (LBM) et par la même la création de matériaux de référence certifiés traçables au Système International (SI) étaient donc des étapes nécessaires. Par ailleurs, l'exigence d'accréditation des LBM est venue confirmée l'accélération de ce processus.
\end{abstract}

Le coût de la mise en place de la métrologie fait oublier parfois, voire trop souvent, la possibilité de limiter des dépenses futures liées à une mauvaise gestion des appareils, à des ré-analyses systématiques, etc. Le coût de la métrologie, sans être négligeable, est à limiter. Il ne s'agit pas de faire de la sur-qualité et d'utiliser des moyens de mesures ultra-performants lorsque le besoin n'existe pas. En ce sens, la démarche doit être la plus pragmatique possible. Aujourd'hui, la nécessité de la métrologie dans les LBM fait consensus. Chacun des acteurs commence à bien connaître les principes de la démarche métrologique.

En revanche, la constatation est sans appel, la théorie se heurte à la pratique. De nombreux laboratoires se retrouvent confronter à des questions légitimes :

- Sur quels équipements assurer une gestion métrologique?

- Comment faire lorsqu'il n'existe pas de prestataire accrédité ?

- Pourquoi mettre en place de la métrologie sur des équipements de mesure alors que le contrôle interne me permet de valider mes résultats ?

- Faire intervenir le service Biomédical me permet-il de répondre aux exigences?

Avant de répondre à ces questions ou du moins de proposer des étapes simples pour y arriver un rappel rapide des exigences s'impose.

\section{Rappel des exigences}

Le laboratoire doit, entre autre, avoir :

- défini la criticité des instruments,

- formalisé un planning d'étalonnage et de maintenance préventive,

- identifier les instruments du laboratoire,

- assurer le suivi métrologique des instruments « critiques »,

- assurer la formation et la qualification du personnel.

Pour répondre à ces exigences, il s'agit d'avancer par étapes.

\section{Des étapes simples pour assurer la mise en place de la métrologie}

\subsection{Formation / qualification du personnel et communication}

Pour pouvoir mener à bien un projet, il est nécessaire de savoir qui en est responsable. Plusieurs laboratoires, ou groupement de laboratoires, ont parfois commencé à mettre en place la métrologie sur certains équipements du laboratoire sans former réellement quelqu'un à cette tâche se perdant ainsi dans les actions à effectuer et reléguant alors la métrologie au second plan comme un domaine réservé aux spécialistes. Il n'en est rien, elle fait partie intégrante de tout processus de mesure.

Définir les actions, prioriser les efforts et sensibiliser le personnel concernant la métrologie est une étape essentielle pour que les actions définies puissent être appliquées. De nombreuses recommandations existent d'ailleurs sur le sujet [5], [6], [7], [8].

La définition d'un « responsable métrologie » quelque soit son titre est donc primordiale. Cette affirmation est également valable lorsque l'on décide d'externaliser ses étalonnages, nous allons y revenir.

Comme évoqué en introduction, la métrologie se heurte à nos vieilles habitudes et croyances : pourquoi mettre en place la métrologie? Nous travaillons bien et notre contrôle interne nous permet de prouver cela. Certes, mais outre l'obligation de mettre en place la métrologie, il s'agit de pouvoir identifier les causes des éventuels problèmes lorsque l'on s'aperçoit d'une dérive significative. Par ailleurs, elle permettra une meilleure connaissance des instruments et donc de définir de mieux en mieux son besoin. Une formation pragmatique, ainsi qu'une communication dirigée et adaptée sont donc deux aspects essentiels pour la réussite de la mise en application de la métrologie avec l'adhésion de tous. 


\subsection{Réaliser l'inventaire des instruments}

Que l'on soit un LBM unique, un laboratoire hospitalier ou même un laboratoire multi-sites, réaliser l'inventaire de ces instruments est une priorité.

Cette étape va permettre d'identifier le besoin de renouvellement éventuel de certains appareils, le besoin d'achat d'instruments plus adaptés au besoin, le besoin d'uniformiser la gestion de certains.

Il s'agit premièrement d'inventorier les matériels utilisés au laboratoire avec leur identification et d'en créer une si nécessaire. Pour cette étape, le rôle du «responsable métrologie » est essentiel, notamment si le nombre de sites/secteurs est important. Il aura la charge de regrouper et d'uniformiser les informations provenant des différents sites.

\subsection{Définition de la criticité}

D'après la nouvelle version de la norme ISO 15189, «le laboratoire doit disposer d'une procédure documentée pour l'étalonnage des équipements susceptible d'affecter directement ou indirectement les résultats d'examens. » Le SH REF 02 [9] précise: «Le LBM identifie ses équipements critiques, c'est-à-dire ayant une incidence significative sur l'exactitude et la fiabilité des résultats, employés dans le cadre de la surveillance des conditions de réalisation des examens (ex : balances utilisées pour préparer un réactif, pipettes utilisées pour préparer une solution servant à un étalonnage, étuve intervenant dans une incubation, etc..). Il identifie ainsi les grandeurs mesurées correspondantes (masse, volume, température, ...) ainsi que les exigences métrologiques spécifiées (tolérances et plages d'utilisation selon recommandations fournisseur, bibliographie disponible ou expérience documentée, performances techniques, ...) et les types de raccordement métrologique employés. »

Cette étape de définition des "équipements critiques » est essentielle. En effet, la gestion métrologie sera focalisée sur ces derniers, permettant également d'ajuster le coût de la métrologie au juste besoin et d'éviter la surqualité. Une fois les «équipements critiques » mis en évidence, il s'agira de savoir si la gestion en place satisfait au besoin et/ou de se poser la question de la nécessité de rebus, d'achat de nouveaux matériels ou d'homogénéisation des utilisations.

\subsection{Gestion des équipements}

Remettre en question la gestion des instruments mise en place jusqu'à maintenant est aussi une étape intéressante.

Certains laboratoires surchargeaient très souvent leurs enceintes ne permettant pas forcément de maintenir les tolérances de températures souhaitées. Suite à l'inventaire, leur décision a été l'homogénéisation et le groupement de leurs produits dans une chambre froide, limitant ainsi les déplacements et le nombre d'enceintes critiques.

A l'inverse, certains n'ayant qu'une seule chambre froide, des problèmes récurrents survenaient. La gestion des équipements a donc été modifiée et l'achat d'enceintes a été programmé.

Cette remise en question a permis à de nombreux laboratoires de limiter les déplacements d'équipements, d'homogénéiser les pratiques de leurs différents secteurs (biochimie, hématologie, etc.).

Par ailleurs, la nécessité de mettre en place les exigences métrologiques de la norme a eu un impact significatif au niveau de la rédaction du cahier des charges lors d'achat d'équipements et de prestations. Plus exigeants sur la demande, sensibilisés à l'importance de formaliser les critères techniques (EMT, incertitudes d'étalonnage, capabilité, etc.), plus à l'aise dans la définition du besoin, les LBM ont appris à prendre du recul par rapport aux notices techniques et à être vigilant concernant la rédaction de leur cahier des charges.

\subsection{Internaliser ou externaliser ses étalonnages}

C'est une question récurrente qu'il est nécessaire de développer, le chapitre suivant permet de formaliser cette question.

\section{Internalisation ou externalisation?}

\subsection{Conserver un minimum de compétence en interne, une nécessité}

Cette problématique était d'ailleurs l'objet d'une newsletter du CT2M [10]. Et chaque laboratoire qui doit gérer un parc d'instruments s'est déjà posé ce type de questions :

Dans la mesure où la gestion métrologique prend du temps pourquoi ne pas externaliser cette prestation? Qu'est-ce qui est le moins couteux ? Si tous les étalonnages de mes équipements sont sous-traités, suis-je dégagé de toute responsabilité en métrologie?

Pour répondre correctement à ces questions, il faut se rappeler que la métrologie est un processus composé de plusieurs étapes : cela commence dès la définition et l'expression du besoin, passe par la planification et la réalisation des étalonnages, et le suivi des éventuels soustraitants, et va jusqu'à la bonne utilisation au quotidien des équipements.

Savoir conserver de la compétence en interne est une nécessité, le «responsable métrologie » est d'ailleurs le 
garant de cette connaissance !! Certaines de ces étapes sont critiques et ne peuvent être effectuées que par une personne qui connaît le contexte dans lequel est utilisé l'instrument. Une phrase résume bien cette problématique : "Celui qui sait à quoi sert l'instrument est le seul à savoir quelle métrologie mettre en place." Si tout est externalisé, qui va vérifier la qualité des prestations réalisées par les sous-traitants ? Surtout, qui va vérifier si elles sont et restent adaptées au besoin?

Il ne faut donc pas se tromper d'externalisation, il faut pouvoir conserver des responsabilités en interne, notamment pour la définition du besoin et le contrôle des prestations réalisées. Ainsi, il faut bien différencier la sous-traitance de la «fonction Métrologie », qui n'est pas conseillée, et la sous-traitance de l'étalonnage, qui est plus facilement envisageable.

\subsection{Etalonnage interne ou externe?}

Alors étalonnage interne ou externe ? La question se pose.

L'étalonnage ne représente qu'une étape du processus métrologie. Une fois le besoin bien défini (points d'étalonnage, méthode utilisée, incertitude souhaitée, ...), l'opération d'étalonnage peut tout à fait être confiée à un prestataire externe compétent. Cependant, si le recours à un sous-traitant peut apparaître comme une solution de facilité, la décision d'externaliser vos étalonnages dépend avant tout de votre situation : nombre d'instruments à étalonner, type d'instruments, procédure d'étalonnage, coût, délai d'immobilisation... Une étude approfondie peut être nécessaire avant toute prise de décision.

Des outils ont déjà été développés en ce sens et permis au laboratoire de mettre en évidence tous les coûts possibles en fonction de la solution déterminée: internalisation et/ou externalisation des étalonnages. Le retour d'expérience montre qu'en général les LBM adoptent une solution intermédiaire, un savant mélange d'externalisation et d'internalisation en fonction de leurs budgets et personnels à disposition.

\subsection{Internaliser les étalonnages: avantages et inconvénients}

\section{$\underline{\text { Avantages }}$}

- Adaptation du calendrier d'étalonnage en fonction du planning interne (et non pas celui de ses sous-traitants !) : on pourra choisir d'étalonner et vérifier ses pipettes au mois d'aout (par exemple) lorsqu'elles sont moins utilisées.

- Meilleure maîtrise de la durée d'immobilisation du matériel : pas de délais de transports notamment
- Vérifications ponctuelles possibles si problèmes rencontrés sur un matériel

- Une fois le mode opératoire rédigé et le personnel formé, le coût et le temps consacré sont réduits.

\section{$\underline{\text { Inconvénients }}$}

- Niveaux d'expertise et de moyens importants: des formations $\mathrm{du}$ personnel et le maintien de leur compétence se révèlent souvent indispensables. Par ailleurs, des investissements importants peuvent être nécessaires les premières années : centrale d'acquisition pour la cartographie d'enceintes, balances étalonnées pour les pipettes, tachymètre pour une centrifugeuse,...

- Avoir un personnel formé pour lequel du temps a été libéré pour ces opérations

- Avoir des modes opératoires rédigés et conformes

- Savoir estimer une incertitude d'étalonnage et réaliser l'estimation pour chaque étalonnage et chaque type d'étalonnage réalisé: il faudra former le personnel, rédiger une procédure d'estimation des incertitudes et des fichiers de calcul adaptés.

\subsection{Externaliser les étalonnages : avantages et inconvénients}

\section{$\underline{\text { Avantages }}$}

- Pas de personnel à former

- Pas de mode opératoire à rédiger

- Conseils techniques de la part des sous-traitants, avis d'experts de l'instrument

- Gamme d'étalonnage des prestataires de métrologie souvent plus importante que les possibilités en interne. C'est le cas notamment des micropipettes, car il faudra un investissement matériel très important pour être capable d'étalonner celles qui ont des volumes nominaux inférieurs à $100 \mu \mathrm{l}$

- Pas d'investissements importants

\section{Inconvénients}

- Coûts annuels

- Indisponibilité (parfois de plusieurs semaines) du matériel pendant l'étalonnage: il est même souvent nécessaire de dupliquer le matériel pour permettre de continuer les analyses.

- Pas de savoir-faire en interne 
- Trouver des organismes accrédités : il n'existe par exemple, à ce jour, aucun organisme accrédité pour l'étalonnage et la vérification de certains instruments.

- Gestion des sous-traitants et acheter une prestation de métrologie adaptée à son besoin : nous allons détailler ce point très important dans le paragraphe qui suit.

\subsection{Et le service biomédical dans tout ça ?}

Dans certains hôpitaux, ils existent des services biomédicaux qui interviennent dans les LBM des centres hospitaliers afin de réaliser des opérations de maintenance et de métrologie. Il est impératif que ces derniers fonctionnent conformément aux exigences de l'ISO 17025 [11]. Pour cela, il est nécessaire que le laboratoire et le service biomédical en question travaillent ensemble pour déterminer l'organisation à définir. Les procédures du laboratoire seront à respecter.

Dans ce contexte, la formation et l'information sont nécessaires pour éviter tout dysfonctionnement. Dans les différents accompagnements réalisés, le service biomédical était invité à participer aux groupes de travail pour la mise en place de la métrologie au sein du laboratoire. Son adhésion et sa compréhension des exigences, issues de la norme ISO 15189 concernant la métrologie, sont des conditions sine qua non de la réussite de tout projet d'accréditation dans lequel un tel service intervient.

\subsection{Absence de prestataire !!}

Il y a encore peu de temps, il n'existait aucun prestataire accrédité par le COFRAC pour réaliser l'étalonnage des centrifugeuses. Aujourd'hui des organismes existent, mais sont peu nombreux. Ce cas n'est pas unique, il n'existe pas non plus de prestataire accrédité par le COFRAC pour réaliser l'étalonnage des pipettes en verre double trait sur de petits volumes. Deux solutions s'offrent alors aux LBM qui doivent réaliser des étalonnages des instruments «critiques» lorsque les prestataires accrédités par le COFRAC n'existent pas :

- internaliser la prestation (éléments déjà traités dans le présent document),

- faire appel à un prestataire accrédité par un autre organisme européen membre de l'European Accreditation. Par exemple pour la verrerie de classe $A$, il s'agit souvent d'un laboratoire accrédité par l'organisme allemand DAkkS, anciennement DKD.

\section{Et les automates dans tout ça!!}

Pour pouvoir mettre en place la métrologie proprement dite sur les automates de laboratoire, il faudrait pouvoir réaliser la traçabilité au système d'unités internationales (SI) ou par référence à une constante naturelle ou une méthode de référence. Malgré les travaux du Joint Committee on Traceability in Laboratory Medecine (JCTLM), il est encore aujourd'hui impossible de pouvoir raccorder au sens propre toutes les techniques [4], [12].

A l'impossible nul n'est tenu !! Dans ces conditions, la conduite à tenir pour les laboratoires est toujours la même [13]. Pour répondre aux exigences de la norme, le laboratoire doit formaliser au mieux les éléments de traçabilité présents en précisant s'il utilise :

- le manuel d'utilisation du fournisseur pour les dispositifs médicaux, de diagnostic in vitro (DMDIV),

- des étalons reconnus,

- les résultats de ses circuits d'inter-comparaison,

- $\quad$ les résultats des comparaisons de méthode, etc.

\section{Retour d'expérience}

Les différents accompagnements menés dans les laboratoires permettent de mettre en évidence les bonnes pratiques.

La formation et la communication sont deux atouts majeurs pour commencer à «dédiaboliser» ce qu'il se cache derrière le terme «métrologie ». La prise de conscience de tous les acteurs concernant les écarts possibles entre les caractéristiques théoriques et réelles d'un équipement permet d'éliminer les réticences quant à l'objectif métrologique.

Le plus important pour aboutir sereinement est de prioriser les actions à mettre en place pour répondre aux exigences, et notamment d'agir en premier sur les analyses pour lesquelles le laboratoire s'est engagé dans la démarche d'accréditation. Fort de cette expérience, le laboratoire pourra ensuite étendre la métrologie aux autres secteurs et ainsi adapter les coûts de la métrologie, et améliorer la gestion mise en place. 


\section{Conclusions}

La métrologie est en soi transdisciplinaire et la question initiale «comment l'adapter à la biologie médicale?» peu paraître surprenante, mais c'est bien de cela dont il s'agit. A l'heure actuelle, il est encore vrai que certaines prestations ne sont pas adaptées à la biologie médicale et un grand nombre de matériaux de référence sont encore à réaliser. Pour autant, il est tout à fait possible de mettre en place une fonction métrologie efficace afin de garantir la qualité et la fiabilité des résultats. Pour conclure, il serait possible d'utiliser la fameuse maxime de Galilée, il s'agit de "mesurer ce qui est mesurable et de rendre mesurable ce qui ne l'est pas ». Cette affirmation est toutefois à nuancer, comme nous l'avons spécifié, il ne s'agit pas de mesurer pour mesurer. L'objectif est de mesurer le juste nécessaire pour identifier les causes potentielles d'erreurs, lorsque par exemple le contrôle interne nous indique une dérive du processus de mesure. La métrologie adaptée à la biologie médicale existe donc, à vous maintenant de l'adapter à votre laboratoire.

\section{Partenariat}

Cette publication bénéficie du soutien financier de l'Union Européenne via le fonds européen de développement régional (FEDER).

\section{Références}

[1] NF EN ISO 15189 - Laboratoires de biologie médicale - Exigences particulières concernant la qualité et la compétence.

[2] Annexe santé 2 - Importance économique des analyses de biologie médicale, Rapport du groupe de travail sur la métrologie du futur, Académie des technologies.

[3] Proficiency Testing Performance in US Laboratories Results Reported to the Centers for Medicare \& Medicaid Services, 1994 Through 2006, Howerton et al. - Arch Pathol Lab Med - Vol 134, May 2010.

[4] La métrologie peut-elle être utile en médecine ? Philippe Charlet, Marie-Lise Migueres, Marc Priel 14ème Congrès International de Métrologie, juin 2009

[5] Recommandations pour l'installation dans le laboratoire de la fonction métrologie et de la documentation correspondante (document B), Groupe de travail SFBC, Ann Biol Clin 2004, 62 : 479-86

[6] Recommandations sur la métrologie et la maîtrise de la qualité des équipements critiques, SG6-03, Groupe de travail SFBC, Ann Bio Clin 2013, 71 : 235-256
[7] Guide Bio Qualité, Mise en place de la métrologie en LBM

[8] Guide Technique d'Accréditation en Biologie Médicale, SH GTA 01, révision 00, mai 2011

[9] Recueil des exigences spécifiques pour l'accréditation des laboratoires de biologie médicale, SH REF 02, révision 02, février 2013

[10] La lettre du CT2M: Etalonnage: interne ou externe ? Un choix à adapter pour atteindre des sommets, CT2M, mars 2012

[11] NF EN ISO 17025 - Exigences générales concernant la competence des laboratories d'étalonnage et d'essais.

[12] List of all available certified reference materials, database of higher order reference materials, measurement methods/procedures and services, 11 april 2013, www.bipm.org

[13] Traçabilité des étalons et des étalonnages, SG02-08, Groupe de travail SFBC, Ann Bio Clin 2010, 68 : 295304 